\title{
Time-dependent deformation behaviors of weak sandstones
}

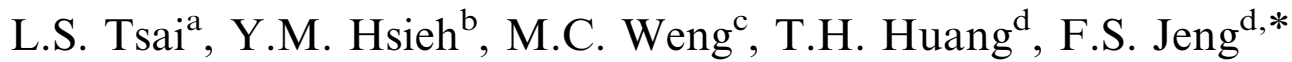 \\ ${ }^{a}$ China Engineering Consultants, Inc., Taipei, Taiwan \\ ${ }^{\mathrm{b}}$ Department of Construction Engineering, National Taiwan University of Science and Technology, Taipei, Taiwan \\ ${ }^{\mathrm{c}}$ Department of Civil and Environmental Engineering, National University of Kaohsiung, Kaohsiung, Taiwan \\ ${ }^{\mathrm{d}}$ Department of Civil Engineering, National Taiwan University, Taipei, Taiwan
}

Received 31 August 2006; received in revised form 5 February 2007; accepted 26 March 2007

Available online 12 June 2007

\begin{abstract}
Time-dependent deformation behavior of rocks has a significant impact on the stability of rock slopes or underground constructions. This paper presents systematic experimental data regarding time-dependent deformation of a typical weak sandstone, known as the Mushan sandstone. The observed deformations are further separated to distinguish elastic and viscoplastic behaviors of the weak sandstone through the use of multi-staged loading-creep-unloading-reloading tests. The stress path is designed to be a purely hydrostatic loading followed by a pure shear, so that the deformations induced by these two types of stresses can be distinguished.

For elastic behavior, although the nonlinear stress-strain relations vary according to the applied hydrostatic stress, these stress-strain relations can be normalized by the applied hydrostatic stress or the bulk modulus and converted into a single consistent stress-strain curve. Inelastic behavior is then obtained by subtracting the elastic deformation from the total deformation. As a result, the characteristics of the viscoplastic deformation are that: (1) the direction of the viscoplastic flow is time independent, and (2) it has a similar direction to the conventionally defined plastic flow. As such, the viscoplastic potential has a similar shape to the plastic potential, but the size of the former changes with time, while the latter has a size that is time independent. Meanwhile, through the calculation of irreversible work, direct evidence of orthogonality between the yield surface and the plastic flow, as well as the viscoplastic flow, is observed. Thus, it is reasonable to assert that the yield surface, the plastic potential, and the viscoplastic potential all have the same geometry. Consequently, the associated flow rules are applicable to modeling the time-dependent deformational behavior of weak sandstones.
\end{abstract}

(C) 2007 Elsevier Ltd. All rights reserved.

Keywords: Weak sandstone; Plastic potential surface; Viscoplastic potential surface; Time dependent; Creep; Yield surface; Associated flow rule

\section{Introduction}

The time-dependent deformation of rocks has a significant impact on the stability of rock slopes and underground structures such as power plants, nuclear waste storage facilities, and tunnels. These engineering projects are susceptible to hazards induced by the time-dependent material behavior during and after construction. In tunneling, "squeezing ground" is one of the typical engineering challenges associated with time-dependent deformation of rocks. After a tunnel excavation and a support installation, the ground may continue to deform as

\footnotetext{
${ }^{*}$ Corresponding author. Tel./fax: + 886223645734.

E-mail address: fsjeng@ntu.edu.tw (F.S. Jeng).
}

time progresses, and may cause severe damage to the tunnel linings and supports if they were not designed to account for time-dependent ground deformation [1-3]. The squeezing ground phenomenon is known to have caused problems in many tunnels built in the sandstone of the Western Foothill Range in Taiwan, and significant remediation costs were required to repair the damage induced by the time-dependent deformations.

Most rocks exhibit various degrees of time-dependent deformation. In order to understand and to characterize the time-dependent deformation, tests within either a very short term (several minutes) to a short term (several hours) were conducted by the rock mechanics laboratory at National Taiwan University [4-6]. These tests and prior research [7-10] concerning the creep behavior of rocks 
show that many different rocks such as rock salt, limestone, granite, chalk, and sandstone, exhibit timedependent viscoplastic deformations. Additionally, irrecoverable and pronounced time-dependent deformations have been observed, even under relatively low stress, for weak rocks such as sandstone, rock salt, and chalk.

It is necessary to understand the general deformation characteristics of rocks before looking into their timedependent behavior. The deformation of rock is conventionally decomposed into elastic and plastic components (which ignores time-dependent behavior in the very short term of loading), or elastic and viscoplastic components (with consideration of time-dependent behavior). However, Perzyna [11] comments that since in practice, it is difficult to separate the plastic and viscous deformations, the separation of deformation into elastic, plastic, and viscous components can be difficult to achieve. Furthermore, a common practice of describing elastic deformations may not be ideal. Elastic deformations are commonly described using isotropic linear elasticity. Maranini and Yamaguchi [10] and Cristescu [12] proposed empirical expressions of the linear elastic modulus, in which the influence of confining pressures on the deformational behavior of granite and rock salt is considered. However, experimental results [13-15] suggest that rocks can exhibit nonlinear elastic deformation, and coupling between shear stress and elastic volumetric strains, e.g. shear dilation or shear contraction, is obvious. Microscopically, the nonlinear elastic deformation characteristics of rocks are possibly related to the opening and closing of the void space and the microcracks in rock during the loading-unloading processes [12]. Most constitutive models for rocks assume that shear stress will not induce elastic volumetric strain, but this assumption does not conform to the experimental results, especially for weak rocks.

Furthermore, the nonlinear characteristics of the elastic deformation should be of concern when separating the elastic and the viscoplastic deformations from the total deformation. Otherwise, the obtained viscoplastic deformation is likely to contain the elastic deformation and not solely the viscoplastic deformation. The incorrectly deduced viscoplastic deformation may inherently lead to an increase in complexity in constitutive modeling, such that the use of a non-associated flow rule becomes necessary to reach agreement between the measured and modeled behavior.

In this paper, we study the nonlinear elastic-viscoplastic deformation characteristics, in conjunction with the shear dilation/contraction for weak sandstones. The detailed description of the deformation behavior was possible as the experiment was designed to unambiguously separate the nonlinear elastic and the viscoplastic deformations. The experiment consists of triaxial tests with pure shear stress paths (to distinguish shear dilation/contraction) and multistaged loading-creep-unloading-reloading procedures (to separate the viscoplastic deformations from elastic ones). Based on this effort, details of plastic flow, viscoplastic flow, the plastic dilation threshold, the viscoplastic creep dilation threshold, and the yield surface of rocks under various stress conditions are obtained.

\section{Methodology}

The Mushan sandstone, which has caused squeezing ground hazards for tunnels in Taiwan, was studied. This sandstone has a void ratio of $14.1 \%$, a dry density of $2.3 \mathrm{~g} / \mathrm{cm}^{3}$, a saturated water content of $5.8 \%$, and an average uniaxial compressive strength of $37.1 \mathrm{MPa}$. Based on microstructure analysis, the percentages of particles, matrix, and voids are $67.5 \%, 18.5 \%$, and $14.1 \%$, with an average particle diameter of $0.72 \mathrm{~mm}$. Mineralogically, the Mushan sandstone consists of $90.7 \%$ quartz, and $9.0 \%$ rock debris, and is thus classified as a lithic greywacke. The oven-dried specimen used for the experiments has a diameter of $55 \mathrm{~mm}$ and a height of $129 \mathrm{~mm}$. The stress was applied by a servo-controlled system, which controlled the variation of stress to be less than $0.05 \mathrm{MPa}$, about $0.2 \%$ of the applied stress.

During the course of conventional triaxial compression (CTC) tests, both confining pressures and shear stresses vary with each increment of loading. As a result, it is difficult to distinguish the effect of shear and volumetric stresses on the volumetric deformations, which include elastic, plastic and creep components. In order to separate the effects of volumetric and shear stresses on the volumetric deformation, the pure shear stress path is used in triaxial tests. Triaxial tests with pure shear stress paths make it possible to separate the effect of shear stresses and volumetric stresses on deformations, and have been adopted in recent years to study the mechanical behavior of rocks in the three-dimensional stress space [13-17]. In triaxial tests with pure shear stress paths, the confining pressure is held constant by decreasing the cell pressure increments to one-half of the increment of the axial stresses $\left(\Delta \sigma_{2}=\Delta \sigma_{3}=-0.5 \Delta \sigma_{1}\right)$. Such a stress path makes it possible to study the effect of shear stresses on the shear and volumetric deformations.

The experimental and test conditions used in this study are summarized in Table 1. These experiments are designed with engineering applicability in mind, thus a confining pressure in the range $20-60 \mathrm{MPa}$ has been chosen. The reproducibility of the experimental results was studied at confining pressures of 20,40 , and $60 \mathrm{MPa}$, and consistent results were observed between different tests under the same test conditions. For the Mushan sandstone, the pure shear triaxial tests under a confining pressure of $60 \mathrm{MPa}$ have a shear strength close to that obtained from the CTC with $20 \mathrm{MPa}$ of confining pressure. The shear strength obtained from pure shear triaxial tests with $60 \mathrm{MPa}$ of confining pressure or CTC with $20 \mathrm{MPa}$ of confining pressure is equivalent to the shear strength with roughly $800 \mathrm{~m}$ of overburden. Therefore, the experimental data should be applicable to a wide rage of engineering projects. 
Table 1

List of triaxial tests and testing conditions

\begin{tabular}{|c|c|c|c|c|}
\hline Test & $\begin{array}{l}\text { Confining } \\
\text { pressure }(\mathrm{MPa})\end{array}$ & $\begin{array}{l}\text { Number of tests without } \\
\text { unloading-reloading cycles }\end{array}$ & $\begin{array}{l}\text { Number of tests with } \\
\text { unloading-reloading cycles }\end{array}$ & Test conditions \\
\hline $\begin{array}{l}\text { Pure shearing stress path } \\
\text { triaxial tests }\end{array}$ & $\begin{array}{l}20 \\
30 \\
40 \\
50 \\
60\end{array}$ & $\begin{array}{l}3 \\
1 \\
1 \\
1 \\
3\end{array}$ & $\begin{array}{l}2 \\
0 \\
2\end{array}$ & $\begin{array}{l}\text { - Loading rate } \\
\text { Hydrostatic stage: } 5 \mathrm{MPa} / \mathrm{min} \\
\text { Shearing stage: } \\
\text { axial: } 16 / 3 \mathrm{MPa} / \mathrm{min} \\
\text { radial: }-8 / 3 \mathrm{MPa} / \mathrm{min}\end{array}$ \\
\hline $\begin{array}{l}\text { Pure shearing stress path } \\
\text { creep tests }\end{array}$ & $\begin{array}{l}20 \\
30 \\
40 \\
50 \\
60\end{array}$ & $\begin{array}{l}2 \\
1 \\
2 \\
1 \\
2\end{array}$ & $\begin{array}{l}1 \\
0 \\
0 \\
0 \\
1\end{array}$ & $\begin{array}{l}\text { - Unloading/reloading tests at } \\
\quad \eta=0.22,0.44,0.66,0.88 \\
\text { - } 8 \text { creep stresses at } \\
\quad \eta=0.2-0.95 \\
\text { - Creep time, } t_{\mathrm{c}} \text { : } \\
\quad \eta=0.2-0.60, t_{\mathrm{c}}=6 \mathrm{~h} \\
\quad \eta=0.6-0.80, t_{\mathrm{c}}=12 \mathrm{~h} \\
\quad \eta=0.8-0.95, t_{\mathrm{c}}=24 \mathrm{~h} \\
\text { Note: } \\
1 \eta \text { is shear stress ratio } \\
2 \text { All specimens are dry }\end{array}$ \\
\hline
\end{tabular}

Notes on the test procedures:

Step 1. Hydrostatic loading to a desired confining pressure.

Step 2. Pure shear to a certain shear stress ratio $\eta$.

Step 3. Conduct creep test for a certain period of time $t_{\mathrm{c}}$.

Step 4. Perform optional unloading-reloading cycle to obtain the elastic strain.

Step 5. Pure shear to a higher level and repeat steps 3-5 until the specimen fails.

To measure the deformation of the specimen, strain gauges of $20 \mathrm{~mm}$ in length, $120 \Omega$ in electrical resistance, maximum measurable strain of $2 \%$, working temperature between -20 and $80^{\circ} \mathrm{C}$, and a measurement accuracy of $0.85\left[(\mathrm{~mm} / \mathrm{m}) /{ }^{\circ} \mathrm{C}\right]$ were directly attached to the surface of the specimen inside the membrane. In order to connect these strain gauges to the outside of the membrane, we made small openings on the membrane, connected wires through these openings, and then sealed the opening with silicon (Fig. 1). This method has been validated by checking for the presence of cell fluid inside the membrane after the completion of experiments. Cell fluid was not found inside the membrane, thus there was no oil leakage into the specimen. Two full bridges are formed by the attached strain gauges to measure axial $\left(\varepsilon_{1}\right)$ and circumferential strains $\left(\varepsilon_{2}=\varepsilon_{3}\right)$. Finally, the volumetric strain $\varepsilon_{v}=$ $I_{1}^{\prime}=\varepsilon_{k k}$ and the deviatoric shear strain $2 \sqrt{J_{2}^{\prime}}$ (where $I_{1}^{\prime}$ is the first strain invariant; and $J_{2}^{\prime}$ is the second deviatoric strain invariant) of the specimen can be measured and recorded. The procedure was further validated by comparing the measured deformation to the one measured by liner variable differential transformer. Satisfactory agreement between these two measurements was obtained.

For the hydrostatic compression stage of loading, experimental results show that the creep volumetric strain

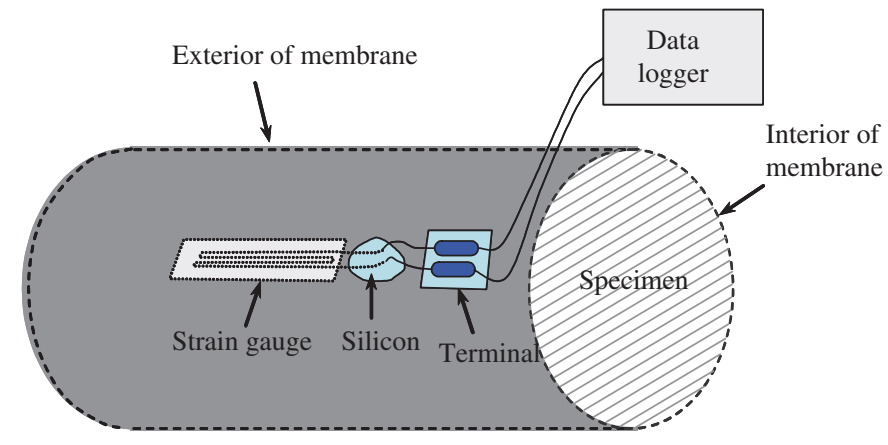

step 1 : attach strain gauge onto the surface of specimen

step 2: Wrap the specimen with membrane, and make opening on the membrane.

step 3: Connect lead wires of the strain gauge through the opening on the membrane to the terminal.

step 4 : Attach the terminal to the exterior of the membrane

step 5 : Connect wires from the data logger to the terminal.

Fig. 1. Schematic illustration of procedures for measuring deformations of the specimen.

monotonically decreases while the confining pressure increases, suggesting that the specimen approaches a stable condition under a greater compression. The creep volume 
strain induced by hydrostatic stress $\left(p=I_{1} / 3=\sigma_{k k} / 3\right)$ is much smaller than that induced by shear stress. Therefore, the creep deformations induced by hydrostatic pressure during the shearing stage of loading were ignored so as to focus on the creep deformational behavior under pure shearing. The same set of loading rates for triaxial tests and creep tests (shown in Table 1) was applied to all experiments under a constant room temperature of $25^{\circ} \mathrm{C}$. Multistaged loading-unloading-reloading tests, as shown in Fig. 2, were performed on selected experiments in order to obtain elastic deformations. We recognized that creep could potentially contribute to the "elastic" strain $\varepsilon^{\mathrm{e}}$ and "viscoplastic" strain $\varepsilon_{0 \rightarrow t_{0}}^{v p}$, as shown in Fig. 2, during the unloading-reloading cycle. However, it was found that the influence of this creep is insignificant, and the framework of the traditional plasticity theory is accordingly adopted in this study so that the separated deformation obtained in this research and others can be compared on the same basis. As such, $\varepsilon^{\mathrm{e}}$ and $\varepsilon_{0 \rightarrow t_{0}}^{v p}$ are regarded as elastic and plastic strain, respectively. For creep tests, this work applied the multistaged loadings shown in Fig. 2d, and the stress applied in each stage has a shear stress ratio $\eta=0.2-0.95\left(\eta \equiv \sqrt{J_{2}} / \sqrt{J_{2 f}}\right.$, where $\sqrt{J_{2}}$ is the second deviatoric stress invariant and $\sqrt{J_{2 f}}$ the second deviatoric stress invariant at failure).

The duration of the creep tests is based on work by Cristescu [17], which suggested the use of the stabilization boundary, defined by data points at the end of the primary creep. This concept was adopted to determine the time interval of creep for each stage of creep. As a result, the time to the end of the primary creep was found to be proportional to the shear stress during the shearing phase of the triaxial tests. Therefore, the present authors have used a creep time of $6 \mathrm{~h}$ for $\eta=0.2-0.6,12 \mathrm{~h}$ for $\eta=0.6-0.8$, and $24 \mathrm{~h}$ for $\eta=0.8-0.95$. When the creep tests have reached the designated time period, the next stage of loading/creep is then conducted. Figs. 3 and 4 show the creep tests for different values of $\eta$ under a confining pressure of $20 \mathrm{MPa}$. The authors kept in mind that the test procedure, the multistage creep test, should be easy to conduct in laboratories in order to increase its applicability when creep behavior is being investigated. An overlong creep test cannot fulfill the needs of engineering projects, as only limited time is available to wait for the a

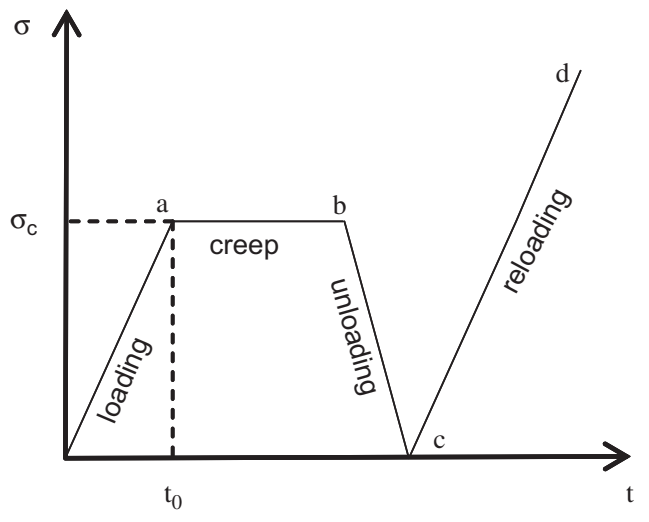

C

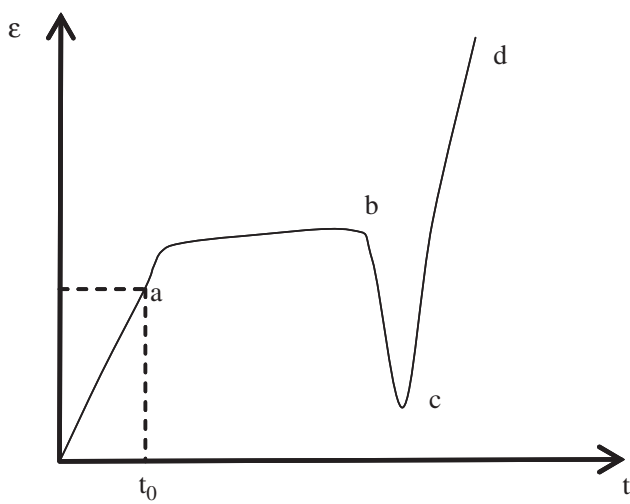

b

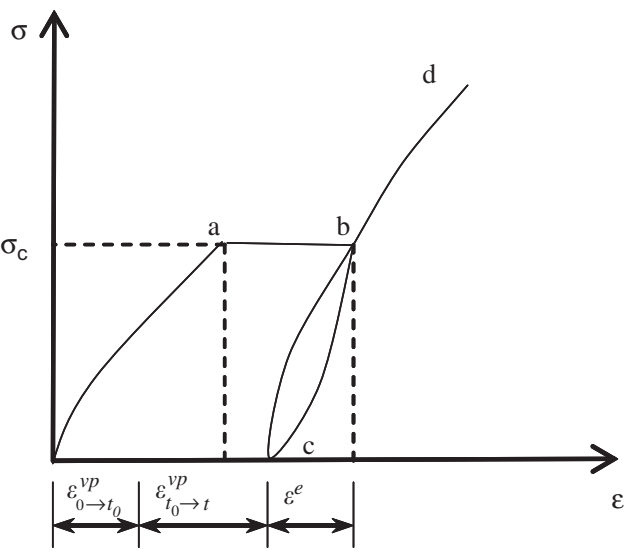

d

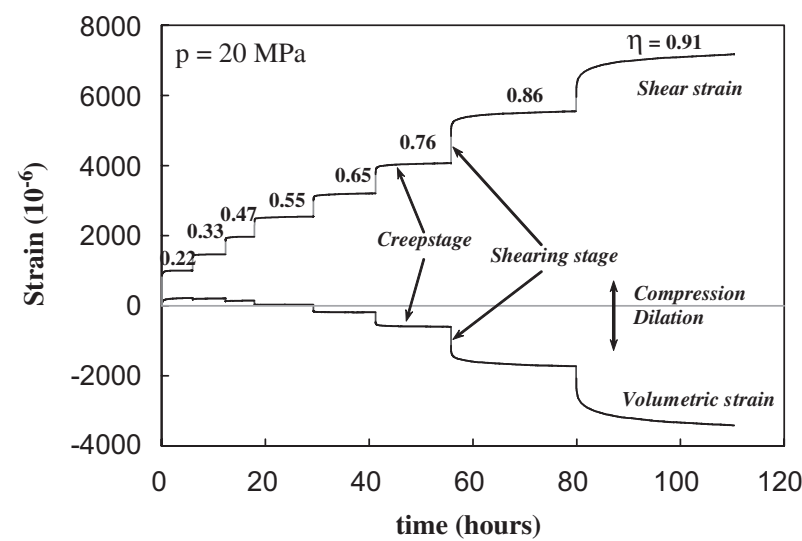

Fig. 2. Schematic illustration of multistep loading creep tests performed: (a) shear stress versus time; (b) shear stress versus axial strain; (c) axial strain versus tie; (d) measured shear and volumetric strain versus time with shear stress ratio of $0.22-0.91$; thin lines represent shearing stage, and bold lines represent creep stage. 


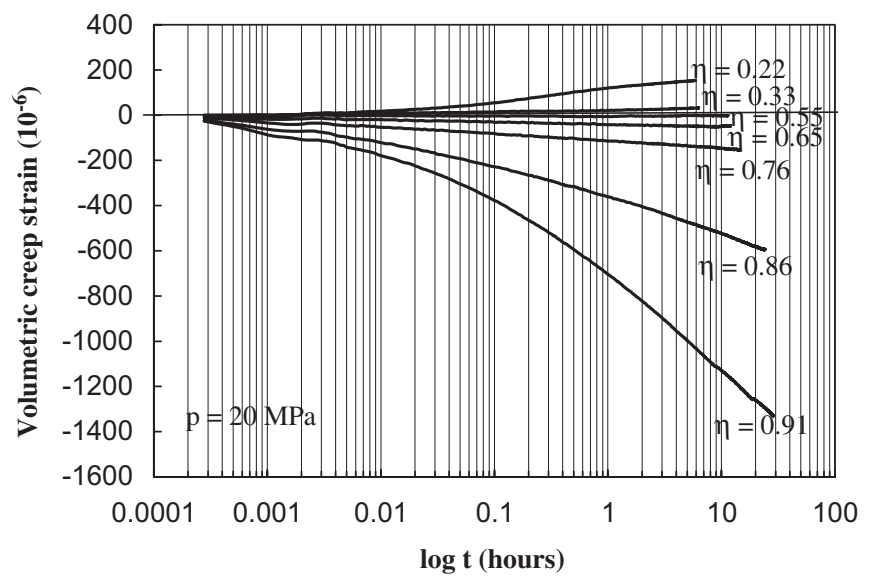

Fig. 3. Measured volumetric creep strain versus time (in log scale) under varying shear stress ratio $\eta$. It is seen that volumetric creep shows compressive behavior at low shear stress ratio $\eta$, and the creep rate decreases with increasing $\eta \cdot \eta=0.55$ serves as the threshold of the transition from compressive to dilative volumetric creep. Further increase of $\eta$ raises the rate of dilative volumetric creep. It should be noted that $\eta$ larger than 0.55 suggests a continuous increase of volumetric creep, and that the material could fail.

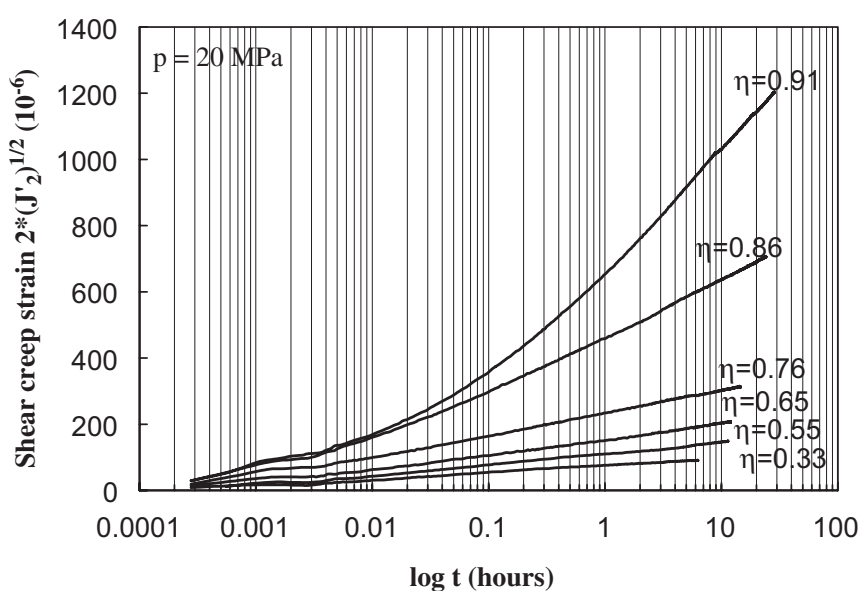

Fig. 4. Measured shear creep strain under varying shear stress ratio $\eta$. Increasing shear creep rate is observed with increasing $\eta$.

experimental results. A few creep tests lasting more than $100 \mathrm{~h}$ had been conducted, and the experimental results showed that the primary creep could have occurred within $24 \mathrm{~h}$. As such, the total test time for one specimen is designed to be about 5 days, while each single step of the creep test can be as long as $24 \mathrm{~h}$ to allow the full development of primary creep.

\section{Elastic deformation behavior}

Based on the small strain assumption and the work done by Perzyna [11], the total strain is the sum of the elastic and the viscoplastic strains, and can be presented as:

$\varepsilon_{i j}^{t}=\varepsilon_{i j}^{\mathrm{e}}+\varepsilon_{i j}^{v p}$, where $\varepsilon_{i j}^{t}$ is the second strain tensor of the total strain, $\varepsilon_{i j}^{\mathrm{e}}$ is the second strain tensor of the elastic component, and $\varepsilon_{i j}^{v p}$ is the second strain tensor of the viscoplastic component.

\subsection{Volumetric stress-elastic volume strain relationships}

In order to separate the total strain into the elastic and inelastic components, the unloading-reloading cycle is used to find the elastic strains. Based on the unloading-reloading test, confining pressure-volumetric strain curves were obtained from several experiments and are shown in Fig. 5. A nonlinear regression curve of the following form was then obtained as

$\varepsilon_{v, p}^{\mathrm{e}}=a_{0} \times \sqrt{\frac{p}{p_{0}}}$.

In Eq. (2), $\varepsilon_{v, p}^{\mathrm{e}}$ is the elastic volume strain induced by hydrostatic stress, $a_{0}$ is a material constant, $p$ is the hydrostatic stress, and $p_{0}=1 \mathrm{MPa}$. For the Mushan sandstone, $a_{0}=1120$ (the bold curve in Fig. 5) is obtained by fitting the experiment data. Based on Eq. (2), it can be seen that the elastic bulk modulus increases nonlinearly with the confining pressure.

\subsection{Elastic volumetric strain induced by shearing}

The shear stress-volumetric strain relationships were obtained from triaxial tests with pure shear stress paths. In this work, three different confining pressures $p=20,40$, and $60 \mathrm{MPa}$ were used to conduct unloading-reloading pure shearing triaxial tests with four different shear stress ratios $\eta=0.22,0.44,0.66$, and 0.88 . Significant shear dilation was observed when $\eta=0.88$, suggesting that the stress state should be close to the yield condition. As a result, unloading-reloading cycles with $\eta=0.22,0.44,0.66$ and $p=20,40$, and $60 \mathrm{MPa}$ (nine different curves) were used for regression analyses to obtain the shear stress-volumetric strain relationships.

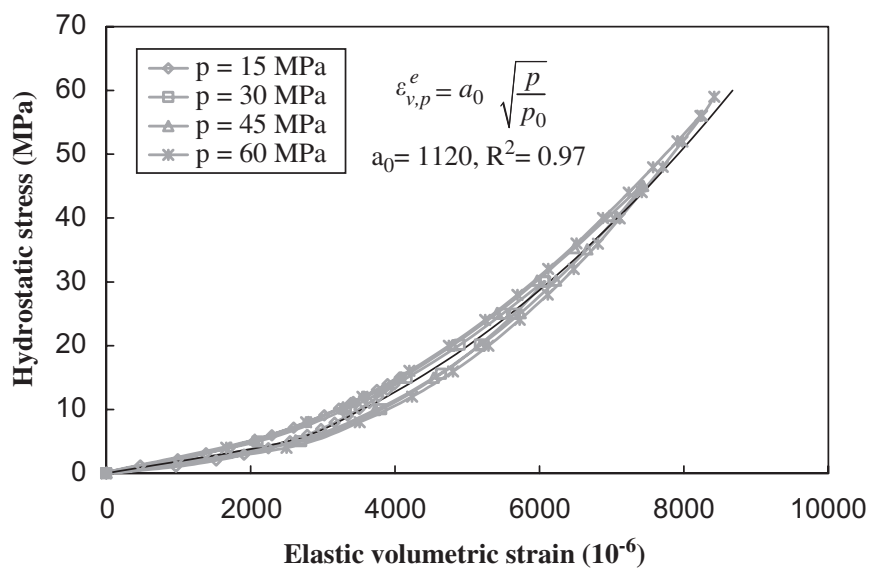

Fig. 5. Measured elastic volumetric strains during hydrostatic loading. The elastic volumetric strain versus confining pressure relationship is concave towards the horizontal axis, suggesting an exponential increase of elastic bulk modulus with increasing confining pressure. 
By normalizing the shear stress $\sqrt{J_{2}}$ with respect to $p$, a regression curve, as shown in Fig. 6, can be expressed as

$\varepsilon_{v, s}^{\mathrm{e}}=-a_{1}\left[\frac{\sqrt{J_{2}}}{p}\right]^{2}$

where $\varepsilon_{v, s}^{\mathrm{e}}$ is the elastic volume strain induced by the shear stress and $a_{1}$ is a material constant.

In Fig. 6, it can be seen that Eq. (3) with $a_{1}=488$ can closely match nine sets of experimental data observed under different conditions. From these results, it can be seen that pure shearing induces elastic volumetric dilation $\left(\varepsilon_{v, s}^{\mathrm{e}}\right)$ on the unloading-reloading path. Thus, the tested weak sandstone indeed exhibits a nonlinear coupling between the shear stresses and the elastic volumetric strains.

\subsection{Shear stress-elastic shear strain relationship}

By using the sets of data obtained from unloadingreloading during pure shearing triaxial tests, the relationship between shear stress and elastic shear strain can be obtained. Fig. 7a shows the shear stress-elastic shear strain relationships obtained from these nine sets of data. A linear relationship obtained by fitting these data has the following form:

$2 \sqrt{J_{2}^{\prime}}=a_{2} \sqrt{J_{2}}$.

In Eq. (4), $J_{2}^{\prime}$ is the second deviatoric strain invariant and is defined as $J_{2}^{\prime}=(1 / 2) e_{i j} e_{j i}$, where $e_{i j}$ is the deviatoric strain tensor and $a_{2}$ is a material constant.

From these data, it seems that $a_{2}$ is a function of $p$ or $K$ (elastic bulk modulus), and the regression shown in Fig. 7a suggests that a larger confinement results in a larger shear stiffness. This data is further analyzed by normalizing the shear stress $\sqrt{J_{2}}$ with respect to $K$ (by taking the derivative of the regression curve shown in Fig. 5 at the respective

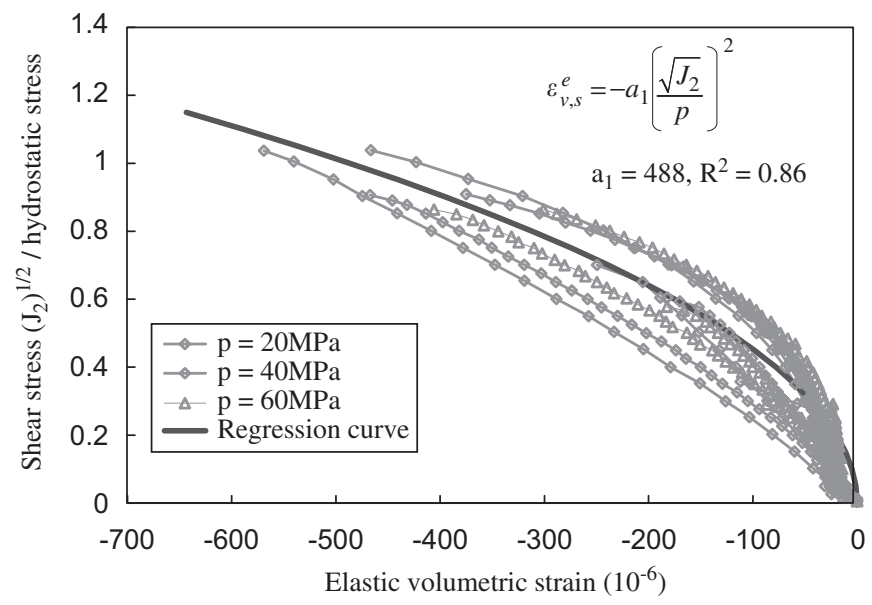

Fig. 6. Normalized elastic volumetric strain versus normalized stress. Pronounced coupling between shear stress and elastic volumetric strain can be observed. This coupling is related not only to shear stress, but also to the confining pressure. a

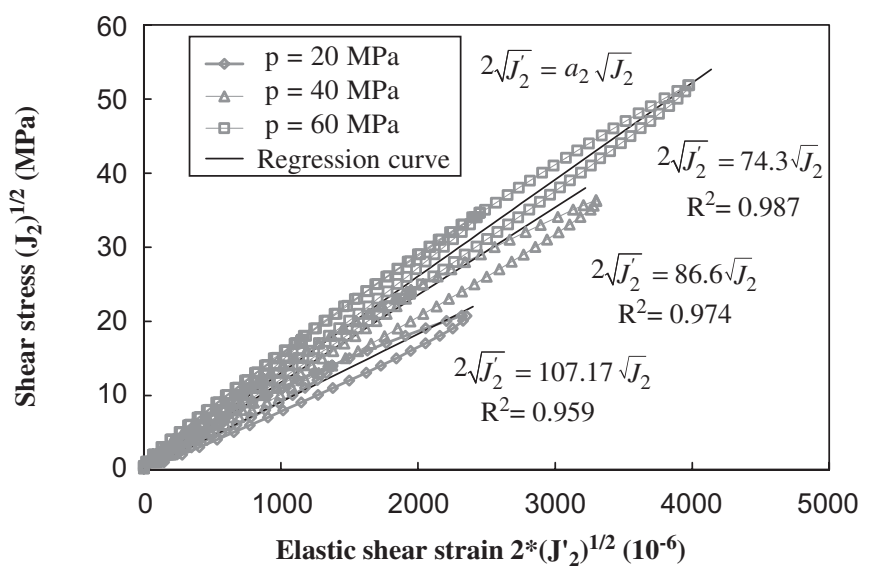

b

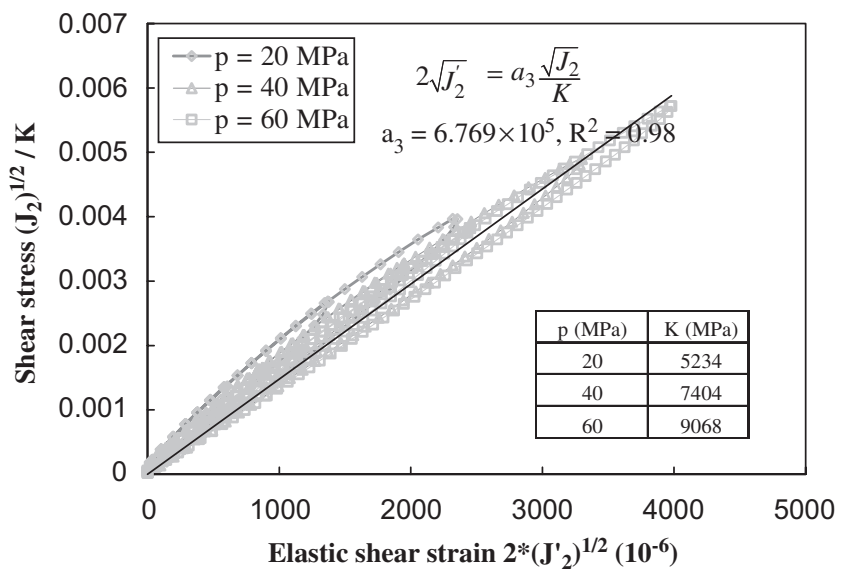

Fig. 7. Measured elastic shear stress-strain during unloading-reloading: (a) measured elastic shear strain versus shear stress; (b) elastic shear strain versus shear stress normalized by bulk modulus.

confining pressures), all these data converge to a single linear relationship, as shown in Fig. $7 b$.

\section{Viscoplastic deformation behavior}

As described in Eq. (1), viscoplastic strains can be computed by subtracting the elastic strains from the total strains. In Section 3, we have obtained the regression equations (Eqs. (2)-(4)) to describe elastic volumetric stress-volumetric strain, shear stress-volume strain, and shear stress-shear strain relationships based on unloading-reloading tests. In this section, the viscoplastic deformation is deduced and its characteristics are then discussed.

The deformations obtained from the pure shearing state of loading were used to calculate the plastic strains (plastic flow) based on methods suggested in Refs. [1, 15]. The deformations obtained from creep tests can be presented as normalized resultant vectors, which are composed of the creep volumetric strain and creep shear strain, to indicate the viscoplastic strain increment (viscoplastic flow). Based on the plasticity theory, we can define $\beta_{1}$ as the angle between the plastic flow and the strain axis (horizontal), 
and $\beta_{2}$ as the angle between the viscoplastic flow and the strain axis as

$\beta_{1}=\tan ^{-1}\left[\frac{\mathrm{d} \gamma^{p}}{\mathrm{~d} \varepsilon_{v}^{p}}\right]$,

$\beta_{2}=\tan ^{-1}\left[\frac{\gamma_{t_{0}-t}^{v \mathrm{p}}}{\varepsilon_{v\left(t_{0}-t\right)}^{v \mathrm{p}}}\right]$,

where $\beta_{1}$ is the plastic flow angle, $\beta_{2}$ is the viscoplastic flow angle, $\mathrm{d} \gamma^{p}$ is the plastic shear strain increment, $\mathrm{d} \varepsilon_{v}^{p}$ is the plastic volumetric strain increment, $\gamma_{\left(t_{0}-t\right)}^{v p}$ is the creep shear strain increment between time $t_{0}$ and $t, \varepsilon_{v\left(t_{0}-t\right)}^{v p}$ is the creep volumetric strain increment between time $t_{0}$ and $t, t_{0}$ is the time at the beginning of creep, and $t$ is an arbitrary time after creep starts.

Fig. 8 shows the variation of $\beta_{2}$ with respect to time for the specimen under $20 \mathrm{MPa}$ of confining pressure. Very small variations of the viscoplastic angle are observed during the experiments. Fig. 9 presents the variation of plastic flow angle $\beta_{1}$ and viscoplastic flow angle $\beta_{2}$ with respect to shear stress ratio $\eta$, and a nearly linear relationship between $\beta_{1}, \beta_{2}$, and $\eta$ is observed. It should be noted that plastic flow angle $\beta=90^{\circ}$ marks the transition from compressive $\left(\beta_{1}<90^{\circ}\right)$ to dilative $\left(\beta_{1}>90^{\circ}\right)$ volumetric plastic behavior. It is apparent from Fig. 9 that under the same shear stress ratio $\eta$, the plastic flow angle $\beta_{1}$ and viscoplastic flow angle $\beta_{2}$ are very close.

As shown in Figs. 8 and 9, it was found that the directions of plastic flow and viscoplastic flows coincide, suggesting similarities between the plastic strain (from pure shearing tests) and the viscoplastic strain (from creep tests). The plastic strain is often obtained from a very short term (a few minutes) loading, in which the time-dependent deformation can still exist and contribute to the deduced "plastic" deformation. The embedment of the viscoplastic deformation in the plastic deformation probably accounts for the similarity between the directions of the plastic flow and the viscoplastic flow. For the sake of convenience, the

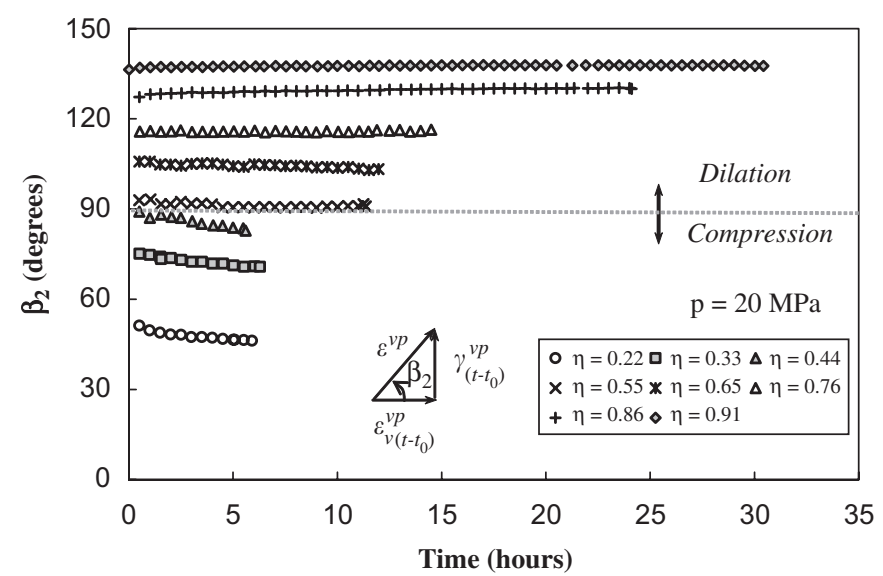

Fig. 8. Measured viscoplastic flow angle $\beta_{2}$ in creep tests. Points shown in the figure correspond to calculated $\beta_{2}$ from measurements done every 30 min under different shear stress ratios.

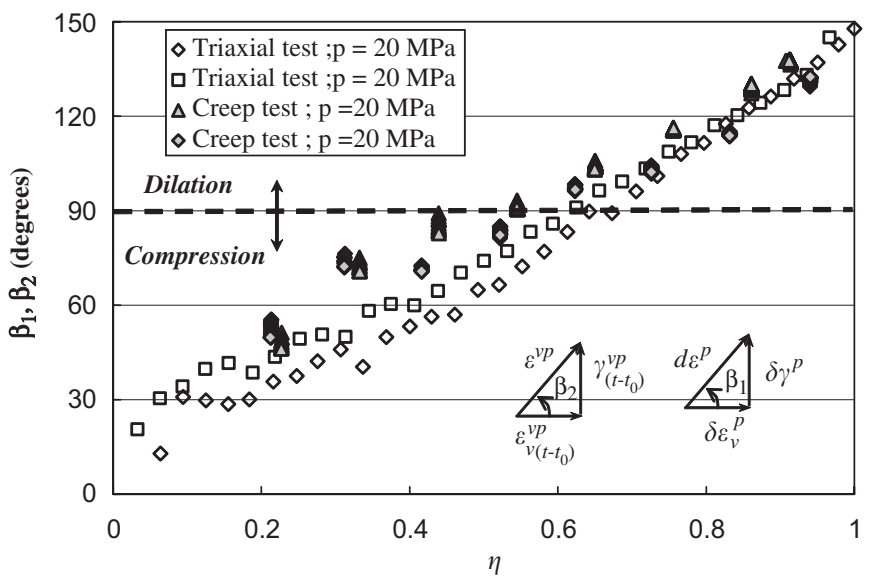

Fig. 9. Measured plastic flow angle $\beta_{1}$ and viscoplastic flow angle $\beta_{2}$ with varying shear stress ratio. It is seen from the figure that $\beta_{1}$ and $\beta_{2}$ are similar under the same shear stress ratio. Therefore, in the stress space of $p$ versus $\sqrt{J_{2}}$, it can be assumed that the plastic flow and viscoplastic flow have the same flow direction under the same stress condition.

very short-term viscoplastic deformation is referred to as the plastic deformation hereinafter.

\subsection{Plastic dilation threshold and viscoplastic creep dilation threshold}

As shown in Fig. 10, both the plastic deformation and the viscoplastic deformation exhibit shear contraction followed by shear dilation when subjected to pure shearing. Therefore, a dilation threshold for both the plastic and the viscoplastic deformations exists. The plastic dilation threshold is a transitional stress state from the volume contraction behavior to the volume dilatation behavior of materials in plastic states under shearing, and is often considered to be associated with the initiation of unstable cracking in sandstones.

Fig. 10 illustrates the relationship between shear stresses and total strains, plastic strains, and viscoplastic strains. Points $\mathrm{A}, \mathrm{B}$ and $\mathrm{C}$ mark the corresponding transition points between the compressive volumetric behavior and the dilative volumetric behavior under one specific confining pressure. Among the thresholds shown in Fig. 10, the dilation thresholds of plastic and viscoplastic deformation (points B and C) are similar and correspond to a greater shear stress level than the threshold of total deformation (point A). It has been observed that a specimen under sustained creep compression, in which the specimen is shear contracted, is less likely to yield. If the initiation of unstable cracks within the rock is related to the shear dilation, then the threshold of plastic or viscoplastic deformation (points $\mathrm{B}$ or $\mathrm{C}$ in Fig. 10, rather than point A) should represent the initiation of unstable crack growth.

Fig. 11 further summarizes the experimental data for these transitional stress states under different confining pressures and shear stress ratios. It is seen that these 


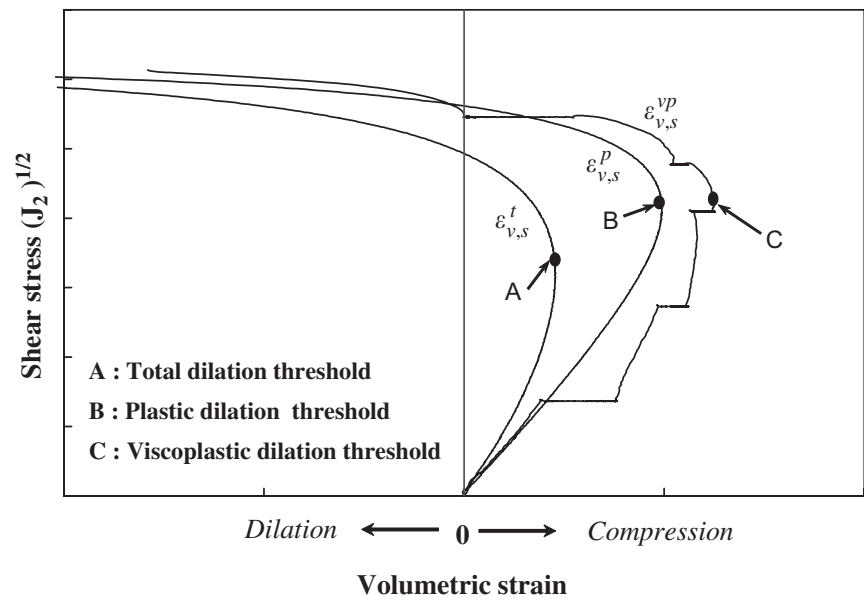

Fig. 10. Dilation thresholds. Points A, B, and C mark the thresholds of three different deformation behaviors. A: dilation threshold of total strain during triaxial shearing; B: dilation threshold of plastic strain during triaxial shearing; C: dilation threshold of time-dependent viscoplastic strain during shear-creep tests.

dilation thresholds are not significantly affected, or only slightly increased by the increase in confining pressure. This result is consistent with the references for sandstones [18-20]. It is also seen that the plastic dilation and the viscoplastic dilation thresholds are still very close to each other for varying hydrostatic stresses.

\subsection{Plastic strain increment and viscoplastic strain}

The plastic strain increment $\mathrm{d} \varepsilon^{p}$ and viscoplastic strain $\varepsilon^{v p}$ are defined as:

$\mathrm{d} \varepsilon^{p}=\sqrt{\left(\mathrm{d} \varepsilon_{v}^{p}\right)^{2}+\left(\mathrm{d} \gamma^{p}\right)^{2}}$

$\varepsilon^{v p}=\sqrt{\left(\varepsilon_{v}^{v p}\right)^{2}+\left(\gamma^{v p}\right)^{2}}$,

where $\mathrm{d} \varepsilon_{v}^{p}$ is the plastic volumetric strain increment, $\mathrm{d} \gamma^{p}$ is the plastic shear strain increment, and $\varepsilon_{v}^{v p}$ and $\gamma^{v p}$ are the viscoplastic volumetric and shear strain increment on the stabilizing boundary, respectively. The plastic strain increment used in this work corresponds to a stress increment of $1 \mathrm{MPa}$ in $\sqrt{J_{2}}$, and Eq. (8) is used to calculate the viscoplastic strain increment.

Fig. 12 shows $\varepsilon^{v p}$ under various values of the shear stress ratio $\eta$ under different confining pressures. It is seen that $\varepsilon^{v p}$ increases with $\eta$ under all confining pressures $p$, and the relationship between $\varepsilon^{v p}$ and $\eta$ appears to be independent of $p$. It is also seen from Fig. 12 that plastic strains can occur even when $\eta$ is very small, suggesting that yielding of weak sandstones occurs at very low shear stress levels, which is very different from the behavior of hard rocks. Fig. 12 also shows a significant decrease in stiffness (inverse of the slope of curves) by a factor of 2-3 under high shear stress ratio $\eta$ for both plastic and viscoplastic behavior.

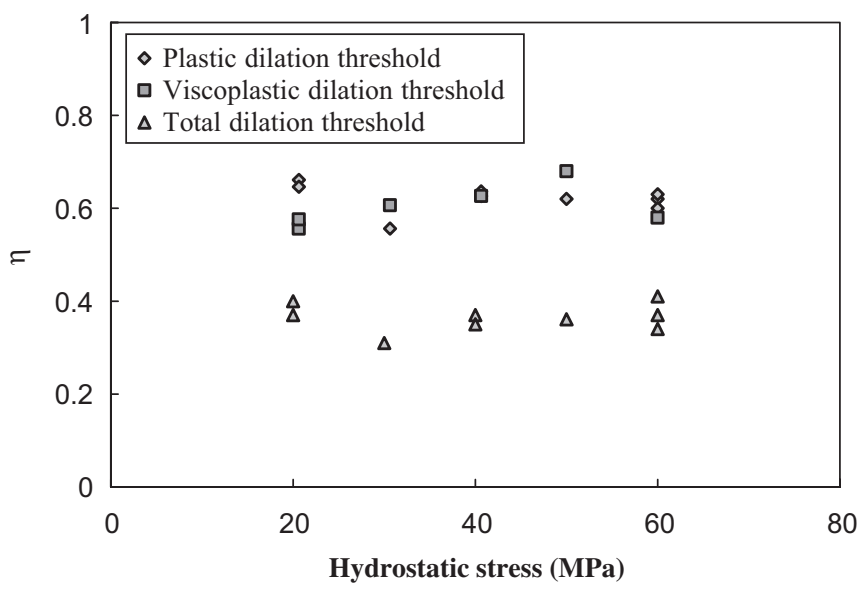

Fig. 11. Measured dilation thresholds under various stress conditions. Similar viscoplastic and plastic dilation thresholds are under the same confining pressure.

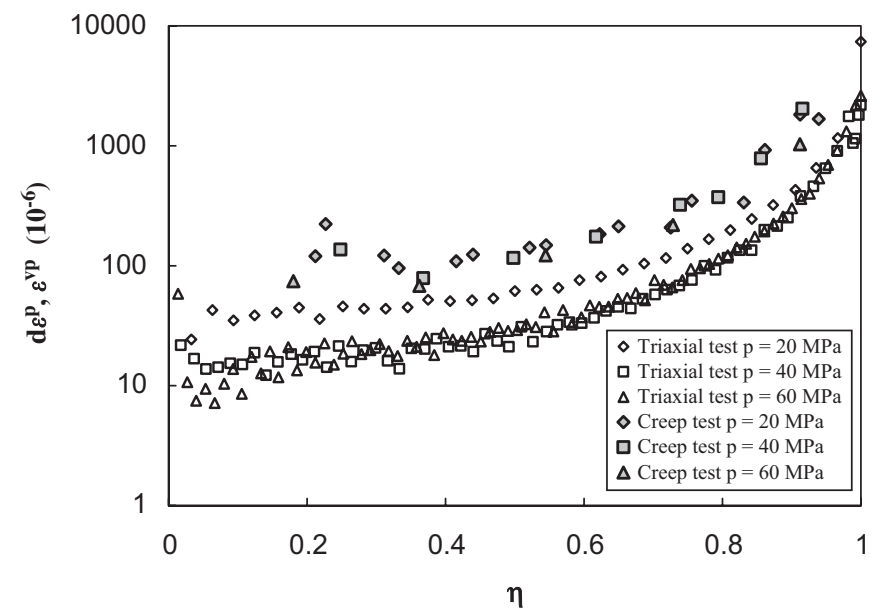

Fig. 12. Plastic strain increments (hollow data points) and viscoplastic strains (solid data points) versus increasing shear stress ratios under various confinements.

\section{Yield, plastic potential, and viscoplastic potential surfaces}

Based on the method described in Section 4, plastic strains were obtained throughout the course of the experiment. Based on the experiments, yielding at small strains was observed, suggesting that the stress state at the beginning of the experiment should be close to the plastic potential surface, which evolves with the increase in shearing. Applying the techniques described in Ref. [15], plastic potential surface can be deduced from plotting the plastic flow, as shown in Fig. 13. It is seen in Fig. 13 that the plastic potential surface before reaching yielding in shear is an elliptical cap. The cap of the plastic potential surface increases its size with increasing stress levels, and coincides with the yield envelope when the material reaches yielding in shear. Similar results are obtained for viscoplastic potential surfaces, suggesting that the weak sandstone has the same surface for both plastic potential 


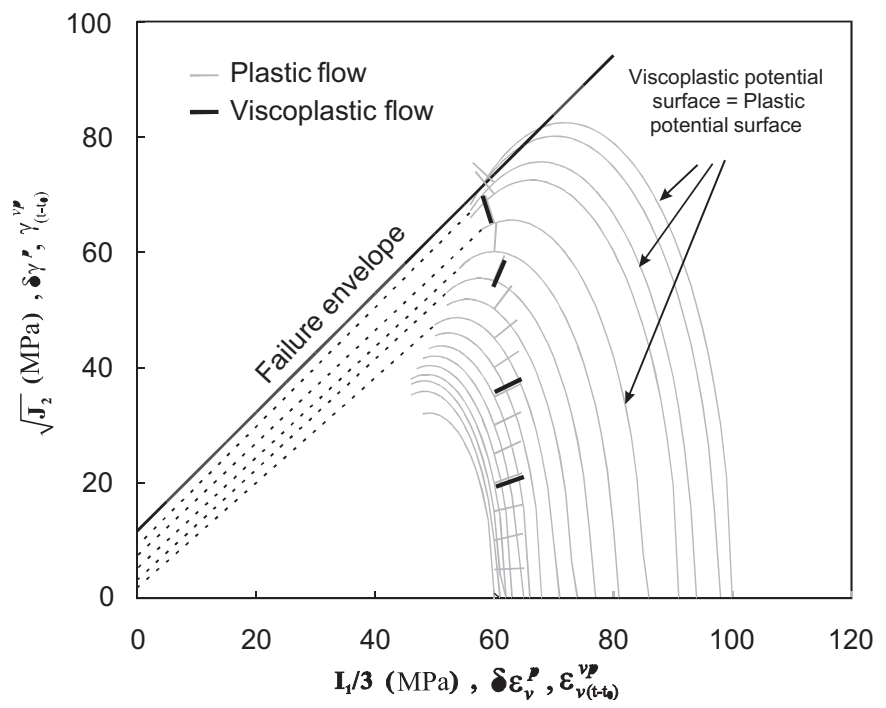

Fig. 13. Illustration of plastic and viscoplastic potentials of sandstones. Nearly identical plastic flow and viscoplastic flow are seen in $p-\sqrt{J_{2}}$ stress space, suggesting identical plastic potential and viscoplastic potential can be assumed after reasonable separation of elastic deformations from total deformations for weak sandstones.

and viscoplastic potential by separating the appropriate elastic deformations from the total deformations.

Under the assumption of homogeneous, isotropic, and small strain, the yield function $H\left(p, \sqrt{J_{2}}\right)$ can be written as $[7,17]$

$H\left(p, \sqrt{J_{2}}\right) \equiv H_{H}(p)+H_{D}\left(p, \sqrt{J_{2}}\right)$,

where $H_{\mathrm{H}}$ is yield function under hydrostatic compression, and $H_{\mathrm{D}}$ is the yield function under pure shearing. In Eq. (9), $H_{\mathrm{H}}$ is controlled by hydrostatic compression, and $H_{\mathrm{D}}$ is controlled by pure shearing. On the stabilization boundary, the value of the yield function equals the irreversible stress work, $W^{I}(t)$. This is because the irreversible strain increment and stress increment are both zero on the stabilization boundary, and the material is in an elastic condition inside and on this boundary. Thus, the yield surface can be defined using $W^{I}(t)$. Cristescu $[7,17]$ suggested that the irreversible stress work $W^{I}(t)$ can be expressed as

$$
\begin{aligned}
W^{I}(t) & \equiv W_{H}^{I}(t)+W_{D}^{I}(t) \\
& =\int_{0}^{T_{H}} p(t) \dot{\varepsilon}_{v}^{I}(t) \mathrm{d} t+\int_{T_{H}}^{T} \sigma^{\prime}(t) \cdot \dot{\varepsilon}^{\prime I}(t) \mathrm{d} t,
\end{aligned}
$$

where $W_{H}^{I}(t)$ is the irreversible stress work under hydrostatic compression, $W_{D}^{I}(t)$ is the irreversible stress work under pure shearing condition, $p$ is the hydrostatic compression, $\sigma^{\prime}$ is the deviatoric stress, $\dot{\varepsilon}_{v}^{I}$ is the irreversible volumetric strain increment during the hydrostatic compression, $\dot{\varepsilon}^{\prime I}$ is the irreversible strain increment and is a general term which can involve the volumetric and the deviatoric components during deviatoric shearing, $T_{\mathrm{H}}$ is the time at the end of hydrostatic compression, and $T$ is the time corresponding to a certain time during pure shearing.
If there is no coupling between the shear stress and elastic volumetric strain, then the volumetric strain during pure shearing can be treated as irrecoverable deformation [17]. Nevertheless, based on the experimental evidence shown in Section 4.1, coupling between the shearing and volumetric deformations is evident for weak sandstone. The elastic bulk modulus is related not only to the confining pressure, but also to the shear stress state. Therefore, for both weak sandstones and materials, the irreversible stress work can be further distinguished as

$$
\begin{aligned}
& H_{H}(p)=W_{H}^{I}(t)=\int_{0}^{T_{H}} p(t) \dot{\varepsilon}_{v}^{I}(t) \mathrm{d} t \\
& \begin{aligned}
H_{D}\left(p, \sqrt{J_{2}}\right) & =W_{D}^{I}(t)=\int_{T_{H}}^{T} \sigma^{\prime}(t) \cdot \dot{\varepsilon}^{I^{\prime}}(t) \mathrm{d} t, \\
& =\int_{T_{H}}^{T} \sqrt{J_{2}}(t) \dot{\gamma}^{I}(t) \mathrm{d} t+p \int_{T_{H}}^{T} \dot{\varepsilon}_{v}^{I^{\prime}}(t) \mathrm{d} t .
\end{aligned}
\end{aligned}
$$

In Eq. (12), $\dot{\gamma}^{I}$ is the irreversible shearing strain increment under deviatoric shearing, and $\dot{\varepsilon}_{v}^{I^{\prime}}$ is the irreversible volumetric strain increment in shear. Remarkably, the elastic volumetric strain has been removed to obtain these two increments. As will be shown later, this removal of elastic volumetric strain enables a simple associated viscoplastic flow rule. Fig. 14 shows the variation of $W_{H}^{I}$ versus confining pressure $p$ during hydrostatic loading for the weak sandstone with four different final confining pressures. It is seen that $W_{H}^{I}$ increases with increasing $p$, suggesting that sandstones exhibit work-hardening behavior.

Fig. 15 shows $W_{D}^{I}$ versus shear stress $\sqrt{J_{2}}$ under confining pressures of $20,30,40,50$, and $60 \mathrm{MPa}$. An exponential increase of $W_{D}^{I}$ with $\sqrt{J_{2}}$ is seen regardless of the confining pressures, suggesting that sandstones also exhibit work-hardening during pure shearing. This is because each unit increase of the shear stress results in exponentially increasing strain increments due to plasticity, thus a larger increment of $W_{D}^{I}$ is achieved.

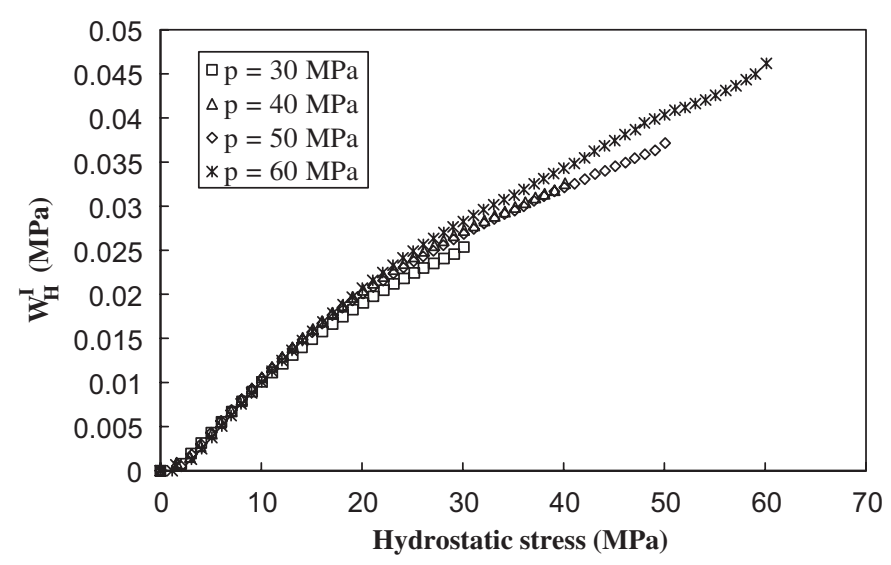

Fig. 14. Measured irreversible volumetric stress work during hydrostatic loading. 


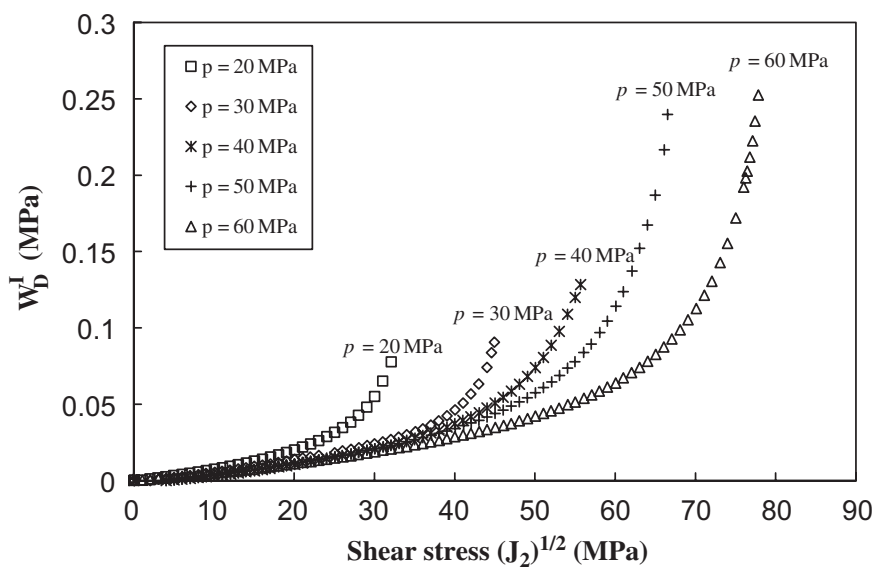

Fig. 15. Measured pure shearing irreversible stress work during pure shearing.

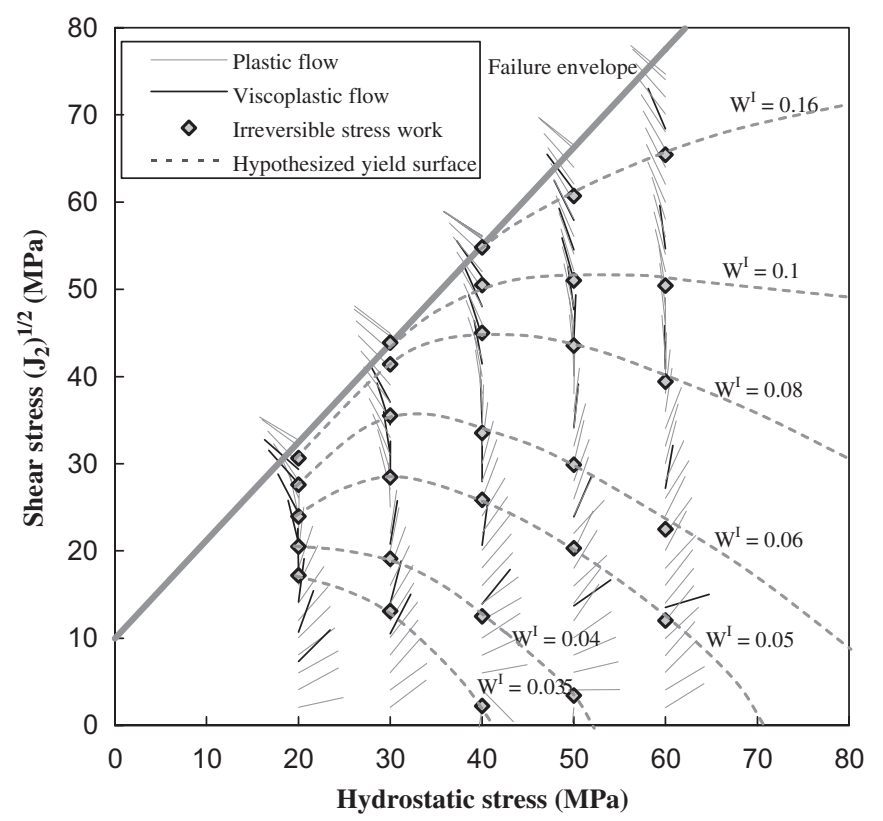

Fig. 16. Derived yield surface, plastic and viscoplastic potentials. Plastic flow and viscoplastic flow are reasonably orthogonal to the yield surface under varying hydrostatic stress, suggesting that the associated flow rule can be applied to model weak sandstones.

By using Eq. (9), along with data in Figs. 14 and 15, the yield locus in Fig. 16 can be obtained by plotting the contours of $W^{I}$ in the stress space. Fig. 16 also shows: (1) the plastic flow direction, (2) the viscoplastic flow direction, (3) the hypothesized yield surface (contours of irreversible stress work), and (4) the failure envelope. It can be seen from Fig. 16 that both the plastic flow and the viscoplastic flow are mostly orthogonal to the yield surfaces, suggesting that both plastic potential and viscoplastic potential take the same form of yield surface, thus associated flow rules can be used when formulating the constitutive models for weak sandstones. However, the plastic potential surface is not necessarily the same as the viscoplastic potential surface, as the plastic potential is time independent, while the viscoplastic potential surface is time dependent. The size of the viscoplastic potential surface will increase as time elapses.

\section{Conclusions}

Details and corresponding insights into the time-dependent deformational behavior of the Mushan sandstone, a typical weak sandstone found in Taiwan, are presented and analyzed based on a series of pure shear stress path triaxial tests and multistage creep tests. For the weak sandstones tested in this investigation, it was found that the elastic behavior, which was used to deduce their plastic or viscoplastic strains, is nonlinear and differs from that of the linear materials. Furthermore, strong coupling between the pure shear stress and the elastic volumetric behavior was also found, and can be described by a regression function normalized by the confining pressure (Eq. (3)).

It was found that the plastic dilation threshold of the investigated weak sandstone is, in fact, quite close to the viscoplastic dilation threshold. This is quite different from results based on the assumption that there is no shear dilatancy and no shear contraction. Such assumptions lead to a significantly lower threshold for plastic dilation than for creep dilation. By introducing the shear dilation/ contraction, it is found that the plastic dilation threshold is close to the viscoplastic dilation threshold, and this threshold can be regarded as the initiation of unstable cracks inside the rock material, suggesting the initiation of micro-crack opening inside the specimen. It is also concluded that the direction of the plastic flow is similar to that of the viscoplastic flow, suggesting that the plastic potentials coincide with the viscoplastic potentials. The experimental data shows that the plastic strain and viscoplastic strain increments are orthogonal to the yield surface, which implies that the associated flow rule in plasticity theory is applicable. In other words, the yield surface, the plastic potential, and the viscoplastic potential may take the same form.

Consequently, separations of the coupling between the pure shearing and the volumetric deformations could potentially simplify the formulation of the constitutive behavior of sandstone materials. Conventional constitutive models that ignore this coupling or assume linear relationships between the pure shearing and volumetric behavior are unable to describe the plastic and viscoplastic behaviors in a more precise manner, and require complex formulations, such as the nonassociated flow rule, in order to achieve reasonable modeling capabilities. Based on the findings presented in this work, the coupling between pure shearing and volumetric deformations should be taken into account when formulating a constitutive model for weak sandstones. Consideration of this coupling allows the use of associated flow rules to describe time-dependent behavior, and thus greatly simplifies the formulation of the constitutive models 


\section{Acknowledgments}

The research was partly supported by the National Science Council of Taiwan (Grant nos. NSC-92-2211-E002-047and NSC 93-2211-E002-047). The authors wish to thank the two anonymous reviewers and the editor for their kind advice, which has significantly enhanced the soundness of this paper.

\section{References}

[1] Jeng FS, Weng MC, Huang TH, Lin ML. Deformational characteristics of weak sandstone and impact to tunnel deformation. Tunnell Undergr Space Tech 2002;17:263-74.

[2] Jeng FS, Huang TH. Time dependent behavior of weak sandstone on Mushan Formation. In: Proceedings of the 13th Southeast Asian Geotech conference. Taipei, Taiwan: Public Construction Commission; 1998. p. 75-80.

[3] Jeng FS, Lin ML, Huang TH. Study of the geological barriers of the tunnels in Northern Taiwan Research Report. Taipei: Ministry of Transportation, 1996. [in Chinese].

[4] Jeng FS, Ju GT, Huang TH. Properties of some weak rock in Taiwan In: Proceedings of the Taiwan rock engineering symposium. Jhongli, Taiwan: National Central University; 1994. p. 259-67 [in Chinese]

[5] Jeng FS, Lee IT, Huang TH. Deterioration of weak rock induced by wetting process In: Proceedings of the Taiwan rock engineering symposium. Taipei, Taiwan: National Taiwan University; 1996. p. 373-82 [in Chinese].

[6] Tsai LS, Jeng FS, Lin ML. Water effects and creep behavior of sandstone weakening. In: Proceedings of the 16th KKCNN symposium on civil engineering. Gyeongiu, Korea: Korea Adv Inst Sci Tech; 2003. p. 575-80.

[7] Cristescu ND, Hunsche U. Time effects in rock mechanics. New York: Wiley; 1998.
[8] Ladanyi B. Time-dependent response of rock around tunnels. In: Hudson JA, editor. Comprehensive rock engineering, vol. 2. Oxford: Pergamon Press; 1993. p. 77-112.

[9] Dahou A, Shao JF, Bederiat M. Experimental and numerical investigations on transient creep of porous chalk. Mech Mater 1995; 21:147-58

[10] Maranini E, Yamaguchi T. A non-associated viscoplastic model for the behavior of granite in triaxial compression. Mech Mater 2001; 33:283-93.

[11] Perzyna P. Fundamental problems in viscoplasticity. Adv Appl Mech 1966;9:243-377.

[12] Cristescu ND. Elasticity of porous materials. In: Lemaitre J, editor. Handbook of materials behavior models. New York: Academic Press; 2001. p. 71-90.

[13] Bernabe Y, Fryer TD, Shively RM. Experimental observations of the elastic and inelastic behaviour of porous sandstones. Geophys J Int 1994;117:403-18.

[14] Jeng FS, Huang TH. Shear dilatational behavior of weak sandstone. In: Proceedings of the regional symposium on sedimentary rock Engineering. Taipei, Taiwan: Public Construction Commission; 1998. p. 262-7.

[15] Weng MC, Jeng FS, Huang TH, Lin ML. Characterizing the deformation behavior of tertiary sandstones. Int J Rock Mech Min Sci 2005;42:388-401.

[16] Hunsche U, Albrecht H. Results of true triaxial strength tests on rock salt. Eng Fract Mech 1990;35:867-77.

[17] Cristescu ND. Viscoplasticity of geomaterials. In: Cristescu ND, Gioda G, editors. Viscoplastic behaviour of geomaterials. New York: Springer; 1994. p. 103-207.

[18] Zhu W, Wong TF. Shear-enhanced compaction in sandstone under nominally dry and water-saturated conditions. Int J Rock Mech Min Sci 1997;34:372.

[19] Zhu W, Montesi LGJ, Wong TF. Shear-enhanced compaction and permeability reduction: triaxial extension tests on porous sandstone. Mech Mater 1997;25:199-214.

[20] Wu XY, Baud P, Wong TF. Micromechanics of compressive failure and spatial evolution of anisotropic damage in Darley Dale sandstone. Int J Rock Mech Min Sci 2000;37:143-60. 